\title{
La Garantie jeunes en pratique dans les missions locales : une expérimentation encadrée
}

The Garantie jeunes in missions locales, in practice: a supervised experiment

Nicolas Farvaque et Carole Tuchszirer

\section{(2) OpenEdition}

Journals

Édition électronique

URL : http://journals.openedition.org/travailemploi/7831

DOI : 10.4000/travailemploi.7831

ISSN : 1775-416X

Éditeur

DARES - Ministère du Travail

Édition imprimée

Date de publication : 1 janvier 2018

Pagination : $15-40$

ISSN : 0224-4365

Référence électronique

Nicolas Farvaque et Carole Tuchszirer, "La Garantie jeunes en pratique dans les missions locales : une expérimentation encadrée », Travail et Emploi [En ligne], 153 | janvier-mars 2018, mis en ligne le 11 juillet 2019, consulté le 05 septembre 2019. URL : http://journals.openedition.org/travailemploi/7831 ; DOI : 10.4000/travailemploi.7831 


\title{
La Garantie jeunes en pratique dans les missions locales : une expérimentation encadrée
}

\author{
Nicolas Farvaque", Carole Tuchszirer**
}

\begin{abstract}
La mise en œuvre de la Garantie jeunes s'est apparentée à une expérimentation encadrée. Les missions locales se sont adaptées à ce nouveau dispositif, en se conformant à des outils de mise en œuvre structurants. Les organisations du travail ont été bouleversées à différents degrés, par le respect des prescriptions nouvelles, notamment l'accueil continu de cohortes au sein des locaux, l'évolution des collectifs de travail avec l'intégration de nouveaux conseillers et le travail en binôme, ou encore la coordination des relations entre conseillers. La Garantie jeunes propose une approche repensée du modèle d'intermédiation des missions locales. La relation aux jeunes a été fortement transformée sous l'effet du dispositif, leur suivi collectif permettant de mieux les connaître. En revanche, la relation aux entreprises n'a, elle, pas été modifiée en profondeur, malgré le principe mis en avant d'une médiation active entre missions locales et employeurs.
\end{abstract}

L 'instauration de la Garantie jeunes et le choix du gouvernement d'en confier la mise Len œuvre aux missions locales s'inscrivent dans une double continuité. Du côté de la politique de l'emploi, ce dispositif expérimental met en évidence le rôle essentiel joué par l'accompagnement des jeunes, quelles qu'en soient les modalités, dans leur processus d'intégration sociale et professionnelle. Depuis la création des missions locales en 1982, la référence à l'accompagnement, qui a été au cœur du projet porté par Bertrand Schwartz pour les jeunes, s'est aujourd'hui imposée à l'ensemble des acteurs du Service public de l'emploi qui en ont fait le cœur de leur modèle d'intervention. Du côté des missions locales, la Garantie jeunes confirme également la place majeure qu'elles occupent désormais au sein des politiques de l'emploi à travers les dispositifs

\footnotetext{
* ORSEU, n.farvaque@orseu.com.

** Cnam (Lise-CNRS), Centre d'études de l'emploi et du travail carole.tuchszirer@1ecnam.net.
} 
mis en œuvre pour le compte des pouvoirs publics. Il s'agit là pour elles d'une évolution plus récente que l'on peut dater du milieu des années $2000^{1}$.

Cette reconnaissance institutionnelle des missions locales dans le paysage des intermédiaires du marché du travail et des politiques d'insertion contribue, en conséquence, à une plus forte visibilité du travail réalisé par les conseillers, notamment du fait des évaluations dont font régulièrement l'objet les dispositifs d'insertion et partant les missions locales. C'est dans ce cadre que s'inscrit cet article, issu de l'évaluation du dispositif, visant à rendre compte de la façon dont les missions locales, des managers aux conseillers, ont déployé la Garantie jeunes sur leur terrain d'intervention.

Nous nous plaçons ici dans la perspective d'une sociologie de la mise en œuvre des politiques de l'emploi (HILL, HUPE, 2002 ; WRIGHT, 2002), interrogeant notamment les décalages qui surviennent entre un cadre défini a priori par les initiateurs du dispositif et sa mise en œuvre sur le terrain. Nous souhaitons plus précisément analyser ici la façon dont le modèle d'intermédiation des missions locales entre les jeunes et les employeurs, repensé par les concepteurs du dispositif notamment sous l'angle de l'accompagnement collectif et de la médiation active, a pu évoluer au contact de sa mise en pratique, ainsi que la façon dont l'épreuve de la mise en œuvre a fait évoluer les missions locales elles-mêmes.

Après avoir retracé l'évolution du modèle d'accompagnement des missions locales en direction des jeunes, nous nous interrogerons sur une dimension originale de ce dispositif dont la conception a reposé sur une démarche expérimentale, une notion dont on analysera la part d'ambiguïté qu'elle a pu susciter auprès des missions locales tant il s'est avéré difficile pour ces structures d'innover dans le cadre d'une expérimentation définie et en partie pilotée par les pouvoirs publics. Nous reviendrons ensuite dans une dernière section sur les modalités pratiques de déploiement de ce dispositif à travers trois de ses principales innovations : le repérage du public visé par la Garantie jeunes, l'accueil par les conseillers de la notion de "médiation active » auprès des entreprises et l'accompagnement collectif délivré aux jeunes.

\section{La place de la Garantie jeunes dans le modèle d'accompagnement et d'intermédiation des missions locales}

\section{Le modèle des missions locales comme intermédiaires de l'insertion}

Nées du rapport commandé à Bertrand Schwartz en 1981, les missions locales (ML par la suite) ont connu au cours de leur histoire de nombreuses inflexions quant à l'orientation de leur référentiel d'activité. L'accompagnement global imaginé à cette

1. Loi de Cohésion sociale dite loi Borloo de 2005, qui intègre officiellement les missions locales dans le Service public de l'emploi. 
époque, pour répondre à la problématique de la jeunesse, reposait sur le pari d'une vision large de l'insertion dans ses dimensions sociale et professionnelle. Le traitement simultané des questions relatives à la famille, au logement, à la santé, à la culture, à la formation, à l'emploi et, plus généralement, à l'accès à la citoyenneté, définit progressivement un territoire professionnel.

Ce travail des conseillers peut s'appuyer sur différents dispositifs ou mesures, à prescrire en fonction du diagnostic posé sur les jeunes, dans une visée de construction d'un parcours d'insertion. Ces outils, dans le domaine de la transition professionnelle (dispositifs de formation, de préqualification, d'évaluation des compétences, de mise à l'emploi, de stage, etc.) mais aussi de l'accompagnement « global » (aides financières d'urgence, soutien en matière de santé ou de logement, etc.), correspondent à des sources de financement diverses. Ces dispositifs intègrent une logique de rationalisation du travail et d'efficacité organisationnelle de plus en plus prégnante, conduisant à des formes de recentrage de l'accompagnement sur les objectifs retenus par les financeurs.

Pour les missions locales, ce recentrage est de plus en plus orienté vers l'accès à l'emploi (FrETEL et al., 2016). Il s'agit d'une évolution qui prend place depuis le milieu des années 2000. Les ML sont officiellement intégrées au Service public de l'emploi (SPE) en 2005 et, à partir de 2007, engagées dans des conventions pluriannuelles d'objectifs (CPO) avec l'État, poussant à un renforcement de la relation aux employeurs. Depuis 2009, les ML sont également prescripteurs de contrats aidés, en relation avec Pôle emploi puis à titre exclusif avec les emplois d'avenir (après 2012). Or, cette relation aux employeurs « ne va pas de soi » pour les ML (ibid.) : la prospection et le placement ne sont pas considérés comme des dimensions premières de leur action, leur accès au volet «employeurs » du marché du travail reste plus complexe et moins direct, en comparaison avec d'autres intermédiaires. Le travail d'intermédiation des missions locales peut donc apparaître comme déséquilibré, avec un accès privilégié aux jeunes, une connaissance spécifique de leurs difficultés et atouts, et une relation aux entreprises plus fluctuante, même si cette dernière n'est pas figée et évolue justement au gré des dispositifs qui en font un objet central. Dans une perspective conventionnaliste, il s'agit pour ces conseillers de réduire l'incertitude qui prévaut lors des recrutements, en offrant aux entreprises une mise en forme des qualités de l'offre de travail (BuREAU, MARCHAL, 2009). Sur des marchés du travail peu qualifiés, des réseaux d'insertion peuvent ainsi travailler la qualification des candidats à l'embauche, ce qui contrecarre la sélectivité habituelle qui peut se faire à leurs dépens. Ceci reste toutefois fragile car dépendant de la force opérante de ce réseau (BARON et al., 1994) et de la confiance que les employeurs placent dans celui-ci.

Le travail d'intermédiation de ces conseillers spécialistes de l'insertion des jeunes, qui interviennent le plus souvent à partir des demandes et des projets de ces derniers, a été comparé à celui de conseillers spécialistes du placement dans les agences Pôle emploi, partant par contraste davantage des besoins des employeurs (CLASQUIN et al., 2000). À la différence de ces derniers, agissant dans un régime visant à ajuster les 
qualités de l'offre de travail à la demande des entreprises, les missions locales opèrent davantage selon un régime de négociation dans lequel les compétences sont définies en situation (Eymard-Duvernay, Marchal, 1994 ; Bureau, Marchal, 2009). Ces « intermédiaires de l'insertion » (BARON et al., 1994) doivent ainsi entrer dans un rapport négocié et donc incertain avec les employeurs. Ils s'appuient pour cela sur divers rapports d'intéressement (EYMARD-DUVERNAY, MARCHAL, 1994), c'est-à-dire la prise en compte de l'intérêt des personnes qu'il s'agit de mobiliser dans le cadre de négociations. La connaissance de proximité (Roche, 2007) des publics jeunes, que l'on pourrait attacher à une grandeur domestique de l'action de ces intermédiaires, correspond à une forme d'intéressement possible des employeurs. Le développement de réseaux de coopération (WuHL, 1996), de dispositifs localisés (Gouzien, 2009) ou de partenariats (SEMENOwICZ, 2017) entre la sphère de l'insertion et la sphère productive - la mission locale et l'entreprise - peut aussi se faire au croisement de plusieurs formes d'intéressement : notamment, une dimension industrielle (sécuriser des recrutements, rechercher du personnel fiable), une dimension civique (comme la mobilisation d'un registre sociétal de la part des employeurs), ou aussi et surtout une dimension marchande relative aux intérêts économiques du recruteur ${ }^{2}$. Dans certains cas, un dispositif peut être actionné dans ces différentes dimensions ; c'est par exemple le cas des Emplois d'avenir pouvant croiser une réduction du coût du travail et un accompagnement du salarié et de l'employeur, notamment en matière de formation professionnelle (FARVAQUE et al., 2017).

\section{Les missions locales encadrées par les dispositifs d'action publique}

Depuis leur création, si la finalité des missions locales a peu changé, il en va donc très différemment de leurs conditions de fonctionnement (Muniglia, THALINEAU, 2012), liées à l'encadrement de plus en plus fort par des dispositifs d'action publique (FARVAQUE, 2004). Le travail attendu des conseillers en insertion a, en conséquence, lui aussi été traversé par de nombreux changements. Le travail est de plus en plus prescrit par en haut, au moyen de cahiers des charges à suivre et d'objectifs à atteindre, impliquant une conduite du changement implicite. Les pratiques des conseillers de ML évoluent dans le cadre des dispositifs d'insertion dont ils ont en charge la mise en œuvre. L'analyse peut alors se porter sur les écarts entre les référentiels créés « d'en haut » et les pratiques réelles constatées « en bas », en insistant notamment sur l'étude des canaux de diffusion des normes et de leur réception (HiLl, Hupe, 2002). Tout dispositif public fait l'objet de traductions et d'interprétations dans sa mise en œuvre au niveau des agences locales. La mission est parfois implicitement confiée ou déléguée à ces magistratures locales (AstiER, 1997) de définir en situation ce qu'est telle politique, qui elle vise, son fonctionnement concret (DuBOIs, 2010). Par exemple,

2. Nous reprenons ici certaines des grandeurs proposées par Luc BoLTANSKI et Laurent THÉvENOT (1991), qui ont fait l'objet de développements spécifiques portant sur les processus d'intermédiation entre institutions et employeurs (Voir notamment BARBIER, EYMARD-DUVERNAY, 1996). 
à la fin des années 1990, les ML appliquent un vaste programme national (baptisé TRACE) correspondant à une logique de suivi intensif des jeunes les plus éloignés de l'emploi. Celui-ci correspond à un recentrage de l'action du réseau sur un objectif chiffré de taux de sortie vers l'emploi durable de $50 \%$. Le fait de recevoir une somme d'argent de la part de l'État pour chaque jeune qui entre dans TRACE et pour chaque sortie dans l'emploi durable a pu faire passer, pour certaines structures, l'obligation de résultats avant l'obligation de moyens, et notamment celle d'un suivi renforcé (KRETzSChMAR, Michiels, 2000). Le pilotage par les résultats conduit ainsi à des mécanismes de sélection des candidats au dispositif. Le public cible, désigné par le gouvernement, ne représente au final qu'un peu plus d'un jeune sur deux (MAS, 2002). Plus récemment, les ML ont été l'opérateur désigné des emplois d'avenir, principal contrat aidé à destination des jeunes entre 2012 et 2017. Les ML ont à produire des régulations locales, parfois seules, parfois collectivement avec d'autres acteurs comme l'administration déconcentrée, pour faire fonctionner le dispositif (GARRAUD, 2000, p. 153). L'atteinte des objectifs fixés à chaque ML, en termes de nombre d'emplois d'avenir signés, a ainsi poussé à assouplir la définition des contreparties censées être imposées aux recruteurs, ou encore à alléger le suivi des jeunes en réduisant les déplacements chez l'employeur, qui figuraient pourtant dans le cahier des charges de l'accompagnement (FARVAQUE et al., 2017). De multiples paramètres viennent donc modifier le sens de la mise en œuvre des dispositifs d'action publique, tenant

\section{ENCADRÉ}

\section{Méthodologie}

L'article est basé sur un rapport d'évaluation réalisé à la demande de la DARES pour le compte du Comité scientifique de la Garantie jeunes (FARVAQUE et al., 2016). L'étude porte sur deux axes principaux : 1) l'analyse de la mise en œuvre locale de ce dispositif et le fonctionnement de ces partenariats multi-acteurs 2) l'évolution des pratiques d'accompagnement au sein des missions locales.

Trois territoires ont été retenus dans trois départements différents. La mission locale A est située dans un territoire de la vague 1, c'est-à-dire entrée dans l'expérimentation du dispositif dès la fin de 2013. C'est une ML de taille moyenne (35 salariés) sur un territoire semi-urbain. En 2015, 190 jeunes sont entrés en GJ pour un objectif de 220 jeunes. La mission locale B, plus petite (18 salariés) et sur un territoire rural, est entrée en vague 2 début 2015 (objectif atteint de 100 entrées). La mission locale C, située dans une métropole, est la plus grande (une centaine de salariés). Également entrée en vague 2, son objectif était de 700 entrées (677 réalisées). Plus d'une soixantaine d'entretiens (tableau en annexe) ont été réalisés auprès des conseillers et des responsables des ML, de leurs partenaires locaux, y compris d'employeurs, ainsi que des observations d'ateliers avec les bénéficiaires de la GJ et de commissions locales d'attribution et de suivi. Des entretiens complémentaires ont été réalisés dans cinq autres ML de ces trois départements pour étudier les différences de mise en œuvre. Le terrain a été réalisé entre novembre 2015 et avril 2016. 
aux modalités d'organisation et à l'assise financière de ces structures (IGAS, 2016), aux trajectoires de ces organisations (FARVAQUE, MESSAOUdI, 2013), aux profils des conseillers (Muniglia, Thalineau, 2012), au contexte local plus ou moins facilitant, que ce soit en termes de fonctionnement du marché du travail ou de qualité des relations partenariales (BERHUET, 2013), etc.

\section{Les innovations de la Garantie jeunes pour le modèle d'intermédiation des missions locales}

La Garantie jeunes (GJ par la suite) introduit de nombreuses innovations tant dans le concept que dans la façon d'organiser le travail des conseillers de mission locale, au regard de l'accompagnement des jeunes et des services proposés aux entreprises. Un premier élément nouveau est l'incorporation d'une allocation monétaire, qui s'inscrit dans la poursuite d'un modèle hexagonal refusant tout revenu d'assistance pour les jeunes de moins de vingt-cinq ans, mais développant diverses formules d'allocation liées à un contrat d'insertion (LimA, 2016). Le second élément fortement repensé concerne l'accompagnement intensif des jeunes, à la fois sous des modes individuels assez classiques et sous des modalités collectives plus inédites. L'organisation du travail évolue puisqu'il s'agit de mettre en place des binômes de conseillers avec un portefeuille de jeunes réduit (cent jeunes par duo). L'accompagnement s'inscrit dans les recommandations européennes : la GJ vise ainsi à « rendre effectif le droit à une première expérience professionnelle ${ }^{3} »$. L'objectif de l'accompagnement dans la GJ est ainsi parcouru par une référence permanente à l'autonomisation ou à l'empowerment des bénéficiaires ${ }^{4}$. Le parcours est fondé sur la mise en action immédiate ${ }^{5}$. L'innovation porte donc ici sur les moyens et le sens de l'accompagnement. Celui-ci « doit rompre avec l'approche linéaire et séquencée traditionnelle du placement par la qualification au profit du savoir-agir du jeune où chaque action en entraîne une autre et dont la clef d'entrée est la mise en situation immédiate par la valorisation des aptitudes et capacités ${ }^{6} »$. Le terme de work first a ainsi pu être utilisé pour soutenir l'idée d'une prise de distance avec la logique train first, qui ferait de la formation un préalable au travail et à l'emploi. Pour mettre en œuvre cette logique centrée sur les mises en immersion, l'offre de services auprès des employeurs est revue. L'idée de la médiation active vise entre autres à susciter des offres d'emploi non formalisées chez ces employeurs par ces immersions. Le principe de la médiation active consiste à se placer en support constant à la relation. Cette approche emprunte beaucoup à la méthodologie «IOD » (Intervention sur l'offre et la demande), visant à dépasser la

3. Rapport du Groupe de travail présidé par E. Wargon et M. Gurgand, mai 2013.

4. Si la notion d'empowerment est utilisée depuis peu, la notion d'autonomie est quant à elle présente depuis les origines ou presque des missions locales. Voir par exemple CLASQUIN et al. (2000, p. 67) : «L'autonomie du public est ici un objectif majeur. » Ces notions font florès en raison de leur très grande perméabilité et indéfinition.

5. Session de formation « démultiplicateurs », module 1, document d'accompagnement « Présentation du déroulé pédagogique », $3 \mathrm{p}$.

6. Session de formation module 1, op. cit. 
seule mise en correspondance, à distance, des parties au recrutement, pour tenter de définir en situation, sur le poste de travail, les attentes respectives (SALOGNON, 2005). L'intermédiaire du marché du travail est vu comme un entremetteur, dont la « professionnalité se situe dans sa capacité à créer une synergie entre les parties du recrutement »(EYMARD-DUVERNAY, MARCHAL, 1997, p. 41).

Derrière ces concepts, le rôle du conseiller est ainsi censé évoluer ; il ou elle n'est pas un « prospecteur-placier » mais un « médiateur », pour reprendre les termes enseignés dans les sessions de formation ${ }^{7}$, avec comme tâches l'animation d'un réseau d'entreprises, l'intervention en appui-conseil auprès des employeurs ${ }^{8}$, etc. Dans ces formations sur la médiation active, le ministère du Travail insiste sur les attentes des employeurs, notamment les TPE/PME, en matière d'aide au recrutement ou encore d'accompagnement dans l'emploi du jeune nouvellement intégré afin d'être dégagé de toute prise en charge des aspects périphériques du travail et de sécuriser la relation ${ }^{9}$. La Garantie jeunes n'est donc pas seulement un dispositif à l'attention des jeunes, elle est censée - pour citer une nouvelle fois le contenu de cette formation ou encore les textes d'instruction - être « autant » une offre de services auprès des entreprises ${ }^{10}$. Ce dispositif est donc censé modifier la nature de l'accompagnement et de l'intermédiation.

\section{Les missions locales face aux innovations de la Garantie jeunes, entre logiques d'expérimentation et de traduction}

\section{Des outils de mise en œuvre de l'expérimentation très structurants}

La GJ a été lancée de façon expérimentale en octobre 2013 avant d'être généralisée en 2017. Il s'agit toutefois d'une expérimentation encadrée par divers outils structurants. Elle s'appuie ainsi sur une doctrine, terme emprunté aux pouvoirs publics, qui peut se lire dans les diverses recommandations et instructions (cahier des charges de l'accompagnement global et intégré, boîte à outils Garantie jeunes ${ }^{11}$, ou encore kit de déploiement $^{12}$ ). Des stages de formation ont été organisés par la DGEFP, contribuant à expliciter cette nouvelle approche de l'accompagnement et de la relation avec les entreprises sous le prisme de la médiation active. Certains salariés de ML (généralement coordinateurs d'équipes GJ) ont été placés sur des fonctions de formateurs, sous une appellation de « démultiplicateur », l'idée étant bien de diffuser le modèle le plus largement possible. D’autres outils tels que des questions/réponses en provenance du

7. Session de formation module 1, op. cit.

8. Rapport Wargon-Gurgand, op. cit., p. 6.

9. DGEFP, Document de présentation de la Garantie jeunes. Réunion d'information du 6 septembre 2013, PPT septembre 2013.

10. Instruction ministérielle relative à la mise en œuvre de la Garantie jeunes sur les territoires pilotes, 11 octobre 2013.

11. Diffusée par la DGEFP en octobre 2013.

12. Réalisé par le SGMAP (secrétariat général à la modernisation de l'action publique) en novembre 2015. 
ministère permettent d'encadrer la mise en œuvre de la mesure. L'entrée successive dans le dispositif de vagues de ML était également censée permettre un apprentissage progressif.

Ces différents outils ont livré des guides aux acteurs locaux, notamment en matière d'organisation ou de méthodes, qui ont souvent été perçus comme des prescriptions et moins comme des indications. C'est notamment le cas de la durée de l'accompagnement collectif initial, en groupe ou en cohorte, censée remobiliser les jeunes avant de laisser place à un accompagnement plus individualisé. Par exemple, la plupart des ML des deux premières vagues se sont basées sur une convention consistant à organiser l'accompagnement collectif sur une durée initiale de six semaines. Or, selon les responsables du ministère, cette durée n'était pas du tout prescriptive. Évoquée au démarrage et testée par les premières missions locales entrées dans le dispositif, cette durée est devenue peu à peu la norme. Le module de la formation DGEFP déployée lors de notre enquête de terrain parle à ce titre du « mythe des six semaines ». Après une phase de respect de cette convention, plusieurs ML avaient fait évoluer leur pratique à ce sujet, en réalisant l'accompagnement sur quatre ou cinq semaines. Certaines ML avaient toutefois dès le départ opté pour des choix différents.

Le contenu de cet accompagnement collectif a lui aussi fait l'objet d'un outillage et d'un encadrement important. Gérer des collectifs de jeunes est nouveau pour les ML. Dans la GJ, l'accompagnement est d'abord réalisé en groupe, alors que l'entretien individualisé est le cœur de métier de ces conseillers (même s'ils gèrent régulièrement des ateliers, il ne s'agit pas du suivi d'un même groupe sur la durée). Il fallait donc équiper les conseillers de repères pour l'action, d'autant plus que l'accompagnement collectif, intervenant au démarrage de la relation contractuelle, est présenté comme une innovation radicale : on lui attribue des effets de remobilisation immédiate du jeune, d'adhésion au projet, de soutien par les pairs. La formation des conseillers insiste sur le caractère émancipateur du travail en groupe. Des méthodes ou bonnes pratiques issues du secteur de la formation continue ont été fournies aux conseillers, comme notamment la démarche dite des compétences fortes, consistant à mettre en avant des compétences génériques issues de la vie quotidienne ou d'expériences antérieures acquises dans l'emploi ou dans toute autre forme d'engagement à caractère associatif : par exemple la minutie, la persévérance, la facilité à effectuer des tâches répétitives, soit une liste totale de treize compétences. Cette méthode d'animation a été reprise dans la plupart des ML. D'autres indications incluses dans la mallette du conseiller ont été plus librement adaptées ou mises à l'écart, par exemple la réalisation collective d'un blason pour chaque cohorte, définissant des valeurs collectives partagées, mais parfois considérée comme une «méthode scolaire » (directeur, ML B).

Plus que d'une véritable démarche expérimentale, fondée sur des innovations locales aux résultats incertains (LATOUR, 2003), la Garantie jeunes s'est apparentée à une logique de pilotes, permettant de retoucher continûment, par vagues successives, le prototype pensé par en haut. Dans les textes, les opérateurs locaux n'étaient pas enjoints à une application uniforme et pouvaient appréhender localement la mise en 
œuvre de la GJ de diverses façons. Mais globalement, les ML de ces premières vagues ont d'abord suivi une logique de conformité au modèle attendu. Pour ce qui est du contenu de cette phase collective, les conseillers GJ de la ML C, rencontrés en entretien collectif, conviennent qu'une première phase a oscillé entre application des consignes et improvisation. Dans un second temps, ils ont pu prendre plus d'initiatives, par exemple créer leurs propres ateliers : " On se met moins la pression sur les objectifs pédagogiques. » L'idée du respect d'un cadre scolaire, comme l'évoque ce terme d'objectifs pédagogiques, souligne toutefois bien une dimension très verticale dans l'application de la mesure.

\section{Une expérimentation pilotée par des indicateurs de performance}

Cette logique de conformité au modèle attendu est renforcée par le rôle central des outils de pilotage. Les finalités repensées de l'accompagnement ne transitent pas uniquement par une succession de recommandations, d'instructions ou de guides de bonnes pratiques, mais aussi par l'élaboration d'indicateurs d'évaluation de la conformité aux objectifs attendus (SABATIER, 1986 ; SABATIER, MAZMANIAN, 1979). Le budget prévu lors de cette expérimentation était de 1600 euros par jeune entré dans le dispositif. Le versement de cette somme est conditionné par l'atteinte de plusieurs objectifs. $70 \%$ du financement dépendent de l'atteinte de l'objectif annuel d'entrées de jeunes en GJ (voir encadré 1), $20 \%$ dépendent de la réussite en termes d'activation du jeune : celui-ci doit soit être en emploi, soit en formation ou en création d'entreprise à la fin des douze mois de la GJ, ou bien avoir été au moins pendant quatrevingt jours - soit pendant un tiers du parcours (quatre mois) - en périodes cumulées d'immersion ${ }^{13}$ en entreprise. $10 \%$ du versement dépendent enfin d'obligations de reporting administratif.

Ces indicateurs participent ainsi d'une logique d'encadrement de l'expérimentation. Les conseillers doivent faire entrer des jeunes dans ce nouveau dispositif, selon des objectifs quantitatifs établis par des moyens statistiques parfois complexes et discutés. Il s'agissait en effet, au préalable, de dénombrer sur chaque territoire le nombre de jeunes Neets vulnérables et pouvant bénéficier d'un accompagnement vers l'emploi. Ce fut un véritable défi pour la statistique que de parvenir à quantifier cette population. Pour le directeur adjoint de la ML C, le chiffre a été fixé « au doigt mouillé ». La responsable locale de la Direccte reconnaît : «Il y a eu un travail réalisé par le ministère du Travail, mais je n'avais pas les résultats. Donc on a fixé les objectifs sans référence statistique. Ça a été : combien vous en voulez ?» Ainsi la catégorie des Neets vulnérables a-t-elle fait l'objet d'une définition ex ante, conduisant à des outils de mesure de la performance, quand on aurait pu l'imaginer davantage comme

13. Le terme d'immersion est volontairement large, il inclut bien entendu tous les contrats de travail, y compris l'intérim, mais aussi les « périodes de mises en situation en milieu professionnel » ou PMSMP. Il s'agit d'un nouveau contrat (qui n'est pas un contrat de travail) correspondant à des périodes d'immersion d'une à deux semaines pendant lesquelles un employeur offre à un demandeur d'emploi une période de découverte de son entreprise et de ses métiers. 
l'objet en soi de l'expérimentation. Il est ainsi demandé aux ML de s'investir sur un nouveau public de jeunes au profil largement indéterminé au moment où on lance l'expérimentation. De son côté, la multiplication des immersions en entreprise n'est pas uniquement mise en avant pour renforcer l' autonomie des jeunes; elle est aussi chiffrée comme un indicateur de performance et vient orienter le travail d'accompagnement dans le sens voulu par l'administration.

\section{Des organisations bouleversées à différents niveaux}

La GJ a dû affronter l'épreuve du réel lors de sa mise en œuvre concrète. Ces structures ont un rapport quasi familier à l'innovation qui fut, dès l'origine, au cœur de leur projet. Il n'y a donc aucune opposition de principe à l'idée d'expérimenter de nouvelles formes d'accompagnement. Les difficultés auxquelles ont été confrontées les ML ont été moins théoriques que pratiques, liées en grande partie aux bouleversements organisationnels que le dispositif a pu induire dans la vie quotidienne des structures. Ils touchent concrètement aux espaces de travail (accueil de cohortes dans des locaux inadaptés), aux collectifs de travail (recrutement et intégration de nouveaux conseillers) et plus globalement à l'organisation du travail (modalités d'échanges, place de la GJ dans la mission locale, etc.).

La ML A appartient à la vague 1 et est donc présente depuis le début de l'expérimentation sans référence antérieure. Sa directrice explique avoir « essuyé les plâtres ». La ML avait imaginé pouvoir se placer dans une logique d'innovation locale et de construction de sa propre Garantie jeunes. Elle estime au contraire se placer dans une logique plus descendante d'application du cahier des charges. La mission locale a d'abord eu à souffrir de l'arrivée tardive des décrets d'application, du cahier des charges et du kit formation de la GJ. Au-delà de cet effet « coup de feu », classique en cas de lancement, souvent médiatisé, d'un nouveau dispositif d'action publique, les responsables avaient anticipé, selon leurs propres informations, une autre organisation du travail dans laquelle l'ensemble des conseillers étaient impliqués dans la GJ (sans conseillers dédiés à ce dispositif). Ceci avait conduit à une prévision différente des ressources à mobiliser :

« Pour nous, il était évident que la Garantie jeunes, c'était un accompagnement aussi qu'on allait intégrer, que l'ensemble des conseillers allaient aussi faire. C'est pour ça qu'on avait imaginé la répartition en fonction des poids du public, la ressource sur le siège, les antennes. [...] Et donc arrive la formation. Et badaboum, on nous dit : "Voilà, la Garantie jeunes, c'est ça ; la Garantie jeunes c'est : un binôme de conseillers dédiés". »

(Directrice adjointe, ML A)

La GJ introduit ainsi une petite révolution dans l'organisation interne de la ML : la GJ ne vient pas se fondre dans l'offre de service existante ; bien au contraire, c'est à cette dernière de s'y adapter. Lors d'un séminaire organisé par l'ensemble des ML 
du département de la ML C, un directeur avance ainsi, par provocation, le thème de la réflexion collective : "Comment intégrer la mission locale à la Garantie jeunes ? ", alors que, pour nombre de conseillers, c'est la question inverse qui était attendue. Là où la GJ aurait dû être transformée par les missions locales, il s'est parfois produit le phénomène inverse d'une transformation des missions locales par la GJ. Dans le cas de la ML A, la GJ est venue percuter de fragiles équilibres organisationnels. Cette structure était déjà dans une phase de changement important puisqu'elle venait à peine de s'installer dans des nouveaux locaux. La GJ est venue remettre en question la nouvelle organisation physique du travail. Le service emploi disposait d'un espace dédié dans ce nouvel emplacement, lui conférant une forte visibilité. Dans un premier temps, devant la nécessité d'accueillir les premières promotions, ce service a cédé son open space à ces cohortes. La ML va ensuite louer un espace supplémentaire à côté de la ML, suite à l'opportunité du départ de l'entreprise qui l'occupait. Situé à dix mètres de la ML, cet espace est désormais entièrement dédié au dispositif GJ. Celui-ci dispose d'une signalétique particulière, lui conférant une identité spécifique. Si le service emploi finit par récupérer son espace de travail antérieur, tout continue de se passer comme si une nouvelle petite entreprise était venue s'installer à l'extérieur des murs, pour délivrer un service spécifique qui ne s'intègre pas directement dans l'offre de service de la ML. C'est également dans une certaine confusion que se met en place le volet RH. Les offres de poste de conseillers GJ ont été ciblées sur le niveau 2 de la classification. Ceci a profondément déstabilisé le collectif de travail (majoritairement positionné sur le niveau 1, inférieur) qui s'est senti d'emblée marginalisé. Sa directrice aura beau revenir sur ce choix en ouvrant le recrutement à des niveaux 1, la méfiance se répand, face à cette « mission locale bis ». Finalement, sur les six postes ouverts, cinq feront l'objet d'un recrutement externe, faute de candidatures internes. D'autres ML à l'instar de la ML B ont cherché à mixer les profils. Le binôme est ainsi composé d'un conseiller insertion de la ML et d'une recrue avec un profil « formation en collectif » pour des publics adultes handicapés. Les recrutements extérieurs auprès de profils de formateurs ont été souvent valorisés, en raison de leur expérience supposée d'accompagnement collectif. Parmi les conseillers de la GJ à la ML C, on trouve ainsi par exemple un ancien formateur en français langue étrangère ou une travailleuse sociale avec une expérience en centre de formation.

La mise à distance des équipes de la GJ, non seulement physique mais également dans les process de travail, révèle les impensés organisationnels de l'expérimentation. Au sein de la ML C, les sept binômes sont tous localisés dans des locaux spécifiques ou dans les antennes. Lors des premiers mois de la mise en œuvre, ces conseillers n'entretiennent que peu de relations de travail avec leurs collègues du siège (c'està-dire l'ensemble des conseillers insertion et emploi). Cela est reconnu autant par ces différents conseillers que du côté de la direction, qui dit avoir pris des mesures pour recréer du lien entre ces services qui ne travaillent pas ensemble : intervention d'un coach, puis création d'un poste de coordination. Cet exemple montre que le management humain n'avait pas été pensé en amont. Ce point n'est pas abordé dans 
les outils de mise en œuvre de la GJ. Bien que cette mesure représente un changement organisationnel conséquent, ces tâches de gestion d'équipe ne sont pas prévues dans les budgets d'accompagnement de la GJ. Cependant, l'intégration de nouveaux collègues dans un collectif, en proportion importante (aux alentours de $15 \%$ des effectifs $^{14}$ ), positionnés sur des tâches nouvelles (accompagnement collectif, gestion d'une allocation monétaire, etc.) et selon des modalités innovantes (travail en binôme notamment), ne peut se faire sans définir des procédures et repenser l'organisation du travail.

La marge d'autonomie des ML peut s'observer dans l'usage de ces moyens financiers pour renforcer les effectifs. Certaines structures ont sciemment sous-doté les équipes de conseillers GJ au démarrage du programme (au risque d'une intensification du travail, de difficultés à planifier les congés, etc.), ou ont au contraire créé des trinômes en lieu et place de binômes (cas d'une ML dans le département de la ML B). D'autres ont dû renforcer leurs équipes en cours de route (création de postes supplémentaires de conseillers ou de personnel administratif en support). La question de l'utilisation de ces fonds ou de fonds internes pour assurer ces frais de personnel, ainsi que ceux relatifs à la coordination et au management des équipes GJ, rend compte du fait que toutes les missions locales ont adopté des modèles différents d'organisation du travail. L'importance des obligations de reporting a conduit la plupart des structures à dédier des postes à ces tâches administratives, financés sur des budgets normalement consacrés à de l'accompagnement. Plusieurs structures du département de la ML B avaient annoncé fin 2015 qu'elles n'accueilleraient pas de nouvelle cohorte en janvier 2016, afin de se consacrer au travail de saisie et de rattraper le retard, ce qui constitue une inversion des priorités (l'administratif primant sur l'accompagnement) rendue nécessaire par les contraintes gestionnaires.

Pendant la phase observée de lancement de la GJ lors des premières vagues, des craintes objectives (distance physique, absence de process et de communication jusqu'alors) et subjectives (représentations biaisées de ce qui se passe dans ces groupes GJ) se sont ainsi cumulées pour créer le sentiment d'une « scission » (conseillère ML B) et d'un changement qui échappe aux équipes de terrain.

En synthèse, les ML ont fait face à de multiples bouleversements organisationnels dans la mise en œuvre de la GJ. La dimension expérimentale a laissé peu de place à des projets innovants, au moins pour ces ML observées faisant partie des premières vagues et soumises à un changement rapide avec peu ou pas de références pour leur apprentissage. Fortement encadrées par divers outils de mise en œuvre, les structures se sont moins inscrites dans des tentatives d'invention de leur propre GJ que dans une démarche de traduction des concepts. La diversité des ML fait néanmoins émerger des façons de faire variées et non uniformes, en fonction de leur trajectoire propre et de leur organisation préalable, tant sur le plan des relations de travail que de l'organisation

14. Un binôme pour 18 salariés à la ML A ; 6 recrues pour une trentaine de salariés à la ML B ; 15 nouveaux conseillers et 2 personnels administratifs pour une centaine de salariés au total à la ML C. 
physique et spatiale. Au-delà de ces aspects organisationnels, la mise en œuvre de la GJ a fait émerger la nécessité de repenser le travail au regard de trois dimensions spécifiques du dispositif : le repérage du public, l'opérationnalisation de la médiation active et la mise en œuvre d'un accompagnement intensif.

\section{Déployer les spécificités nouvelles de la GJ : repérer les jeunes et les accompagner autrement par une médiation avec les entreprises}

\section{La recherche collective et difficile d'un nouveau public}

La cible visée par la GJ, des jeunes Neets, pauvres et vulnérables, représente un changement majeur pour les missions locales, moins par la situation professionnelle de ces jeunes qu'en raison de la prise en compte de critères de vulnérabilité sociale et familiale. Ce ne sont en effet pas seulement les jeunes Neets qu'il fallait repérer, mais - comme le précisent les textes - les jeunes Neets vulnérables. Le critère de vulnérabilité était défini relativement aux situations de rupture familiale ainsi que par rapport aux ressources du foyer. Aller à la rencontre de ces profils de jeunes en situation de rupture, à la fois professionnelle et personnelle, ne va pas de soi pour les ML. Leur référentiel d'insertion vise à accueillir les jeunes au sein de ces structures mais pas à les démarcher. L'éligibilité à la mesure impose de plus de vérifier les ressources parentales, soit l'intégration d'une logique, nouvelle, de travail social.

Cette nouvelle posture de travail consistant à aller au-devant de la demande des jeunes pour les conduire à s'inscrire dans la GJ devait reposer sur la mobilisation de nombreux partenaires : ceux issus du SPE, mais également ceux relevant de la sphère judiciaire et surtout du travail social, dont le métier précisément consiste à repérer les publics ne fréquentant pas spontanément les institutions pouvant leur venir en aide. Au sein des trois départements, le cœur des partenariats établis par les missions locales a été constitué des partenaires institutionnels classiques. Les Direccte, comme ce fut le cas lors de la mise en œuvre des emplois d'avenir depuis 2013, ont joué un rôle central de mobilisation des acteurs (c'est le cas notamment de la ML C) mais aussi parfois un rôle très directif de contrôle du respect du cadre (ML B). Ailleurs, c'est plus directement le préfet qui a été à l'origine de cette dynamique partenariale. Dans le département de la ML A, le préfet a pris en main le dispositif en encadrant fortement la dynamique partenariale impulsée par la mission locale enquêtée pour exercer le contrôle sur les profils de jeunes intégrant la GJ.

Il a été difficile d'élargir le cercle à des nouveaux partenaires. On peut illustrer ces difficultés dans les relations nouées avec les services de la protection judiciaire de la jeunesse (PJJ) et les services pénitentiaires d'insertion et de probation (SPIP). Si quelques SPIP et missions locales se sont rapprochés, estimant que la Garantie jeunes 
et les démarches d'insertion "pouvaient constituer une alternative crédible aux yeux du juge » (ML C), ailleurs c'est la méfiance qui a prédominé, par exemple dans le cas du département $\mathrm{A}$. Le préfet s'est montré très prudent quant à un rapprochement avec les SPIP estimant que « la Garantie jeune n'est pas là pour influencer les juges » ou servir de contrepartie à d'éventuelles réductions de peine. Dans les trois missions locales, des liens se sont établis avec la PJJ ou le SPIP, notamment en matière d'échange d'informations, mais peu de liens forts ont été instaurés à l'occasion de la GJ entre ces univers qui ont une relation aux jeunes bien spécifique. Mais les relations nouées avec les services judiciaires n'ont pas permis d'ouvrir massivement l'accès de la GJ aux jeunes sous main de justice.

Les travailleurs sociaux des associations locales ou du département sont pourtant des acteurs du repérage de publics bien identifiés, avec lesquels les ML ont des coopérations de travail déjà établies. Au-delà des échanges d'information traditionnels, les travailleurs sociaux, en contact quotidien avec des familles en détresse, ne se sont pas portés candidats à la prescription de la GJ, c'est-à-dire à proposer directement la mesure aux jeunes éligibles. C'est un constat qui vaut sur les trois territoires enquêtés. La dimension « insertion professionnelle » de la GJ a pu poser problème à des travailleurs sociaux estimant qu'ils n'avaient pas forcément à reprendre à leur compte une orientation relevant plutôt des attributions du SPE (missions locales, Pôle emploi, IAE). On touche ici à des aspects qui renvoient au métier, à la culture et à la déontologie des professions du social. Le travailleur social ne se vit pas comme un conseiller d'insertion et sa relation au jeune ne peut être dictée par des considérations renvoyant à la finalité des politiques d'emploi. Le principe d'une allocation monétaire perçue en contrepartie d'un engagement ferme du jeune dans le dispositif et la logique du work first a conduit les travailleurs sociaux à se maintenir à l'écart de l'acte de prescription de cette mesure. La peur « de se griller » avec leur public peut aussi expliquer cette position de repli. Ils se sont limités à inviter les jeunes à se rapprocher de la ML, à qui revient au final la charge de la prescription de la GJ. Dans quelques cas extrêmes, évoqués dans une seconde ML du département A, c'est même par principe que les travailleurs sociaux refuseraient de collaborer avec les ML :

« Pour les purs et durs, ils estiment que hormis eux, personne ne sait faire du travail social. [...] On n'a aucune légitimité à leurs yeux, à connaître les choses sur l'histoire familiale, donc chacun se débrouille dans son coin pour avoir les informations qui lui seraient nécessaires pour travailler. Tout le monde n'est pas comme ça $[\ldots]$ mais ça continue d'être un dogme. »

(Directrice, seconde ML enquêtée département A)

Au final, début 2016, les ML étaient dans près de $95 \%$ des cas à l'origine de la prescription de la GJ. La réalité du terrain semble inviter à la prudence quant à une vision mécanique du repérage, qui minore la complexité du travail réalisé par ces différents acteurs locaux aux cultures professionnelles diverses. Les critères de ciblage ne sont pas pour eux les mêmes que ceux de la ML. L'implication active de 
nouveaux acteurs aurait exigé en amont un long travail de sensibilisation aux enjeux de la GJ, d'échange et de mise en commun des référentiels d'intervention de chacune des institutions, pour s'entendre sur la façon d'envisager un travail collectif. La constitution de ces partenariats censés apporter aux ML un vivier de jeunes correspondant aux critères d'éligibilité de la GJ n'a pas eu la portée escomptée. La plupart des jeunes qui ont intégré la GJ connaissaient les missions locales et étaient connus d'elles.

Les trois ML enquêtées se sont du reste livrées à un travail d'investigation avec d'autres acteurs du territoire pour tenter d'accueillir de nouveaux profils de jeunes. Ce travail de repérage a été promu par les instances du ministère, considérant parfois que les ML seraient observées sur leur capacité à faire venir des Neets. La ML C a ainsi recruté un adulte-relais pour faire ce travail d'aller vers les jeunes. Il se considère lui-même comme un « $\operatorname{chasseur}^{15}{ }^{15} .80 \%$ des jeunes qu'il rencontre ainsi lors de ses déambulations dans les quartiers de la ville étaient déjà enregistrés dans les fichiers de la ML. Les 40 à 50 décrocheurs signalés sur le territoire rural de la ML B (via les élus notamment) ont été convoqués. Pour la très grande majorité d'entre eux, ils étaient déjà connus. Sur la ML A, un travail partiel de croisement de fichiers avec des associations de quartier est parvenu au même résultat. Près de $80 \%$ des jeunes fréquentant ces associations avaient déjà fait l'objet d'une inscription à la ML. Ces résultats nous ont souvent été mentionnés pour démontrer aux pouvoirs publics que leur vision de jeunes «dans la nature », coupés de toute relation institutionnelle, correspondrait à une représentation tronquée de la réalité. Cette connaissance préalable des jeunes ne dit bien évidemment rien de l'intensité des contacts que ces jeunes avaient pu nouer avec la ML ou des raisons de leur désaffection, mais en tout état de cause ces structures semblent bien faire partie de l'environnement quotidien de ces jeunes.

\section{La médiation active, une notion difficile à opérationnaliser}

La relation à l'entreprise sous le concept de la médiation active constitue une autre novation d'importance introduite par la GJ. Comme nous l'avons rappelé plus haut, la relation aux employeurs a fortement évolué depuis le milieu des années 2000. Depuis 2005, des embauches ont été réalisées par les ML pour réorienter le profil des conseillers sur une dimension plus économique, afin de constituer des équipes spécialisées sur l'emploi et la relation entreprise. Cette évolution générale a donc permis aux ML d'accueillir favorablement le principe d'une mobilisation des employeurs locaux. La relation aux entreprises occupe désormais une place considérable dans l'offre de services des ML observées. Pourtant, le principe de la médiation active, tel que théorisé

\footnotetext{
15. Le terme de « chasse aux Neets » a ainsi souvent été entendu et connoté péjorativement. Le fait est que dans le même temps, les crédits européens de l'IEJ (Initiative européenne de la jeunesse), contribuant fortement à la GJ, ont aussi financé des actions de formation ou d'accompagnement réalisées par d'autres acteurs (centres de formations, associations, etc.), créant des phénomènes de « captation » des publics ciblés. « Les organismes de formations veulent faire du Neet à tout prix ! Chacun fait sa prospection de façon sauvage » (directrice ML A).
} 
dans la GJ, combinant services aux jeunes et aux entreprises, a eu du mal à se frayer un chemin opérationnel au sein de ces structures.

Si la médiation active convoque l'entreprise dans le processus d'accompagnement des jeunes, elle le fait en effet sur des registres d'action variables. Une certaine confusion a pu être induite dans les esprits quant au rôle dévolu aux entreprises dans la GJ. Ainsi, la place accordée aux immersions, par le biais des PMSMP, contribue à faire de l'entreprise un partenaire voire un intermédiaire, et non plus un client, du SPE. Ce registre se rapproche quelque peu de la vision portée par Bertrand Schwartz (1994) quant au rôle de l'entreprise dans les processus d'insertion des jeunes : à la fois lieu d'orientation professionnelle, par la découverte de nouveaux métiers, et lieu de formation et d'acquisition d'une expérience, faisant l'objet d'un retour et d'une exploitation de la part de la ML. Mais finalement, peu de missions locales ont utilisé les PMSMP dans une optique itérative, visant à construire un projet professionnel par l'acquisition d'expériences et la découverte successive de différents métiers. La place de ces immersions dans le parcours du jeune rend compte d'un usage plus classique de ces mises en situation de travail. La période d'immersion s'est dès lors apparentée dans la majorité des cas rencontrés à de simples stages ou parfois à des périodes d'essai faisant office de pré-embauche, une fois le projet professionnel établi avec la ML. La recherche d'une immersion occupe une place importante dans les premières semaines d'accompagnement collectif. Ces périodes d'immersion peuvent donner lieu dans certaines ML à un bilan pour notamment « repérer si le jeune est fait ou non pour le métier » (CGJ, ML B). Mais pour la plupart des conseillers GJ, soumis à une forte pression temporelle liée au suivi des cohortes, le temps a manqué pour travailler sur ces retours d'expérience. Les bilans effectués de façon plus ou moins régulière n'ont pas toujours permis de tirer pleinement parti de ces phases d'immersion, qui ont eu du mal à remplir des fonctions d'orientation, de formation.

Un deuxième registre d'intervention relève davantage d'une fonction sociale qui attribue à l'entreprise des propriétés susceptibles de lever, en situation de travail, les freins périphériques à l'emploi sans en faire un préalable à l'embauche. Il s'agit là d'une inversion forte par rapport à l'ensemble des dispositifs d'insertion qui entendent traiter les problématiques sociales en amont du recrutement. Cette fonction sociale de l'entreprise, qui parie sur les mises en situation pour faire émerger les difficultés à traiter, sans faire de la levée de ces freins un préalable au retour à l'emploi, s'est révélée délicate à mettre en œuvre. Pour un certain nombre de jeunes, ces freins sont revenus comme une contrainte préalable à lever en amont de l'entreprise voire même en amont de la GJ. Face à des cas individuels de forte vulnérabilité (addictions, jeunes à la rue, etc.), la vision portée par les textes s'est révélée en décalage avec les pratiques habituelles des conseillers de ML. Au sein de la ML A, les problématiques sociales de santé, de logement, d'addiction, sont vues comme prenant le pas sur la dynamique du retour à l'emploi. Confrontés à la présence de jeunes avec de telles vulnérabilités, les conseillers ne parviennent pas à neutraliser ces problèmes qui viendraient remettre en cause la cohérence des cohortes accompagnées. Pour ce type de publics, les responsables de 
cette ML suggèrent de placer un sas en amont de la GJ, afin de lever tout ou partie de ces difficultés. Cette analyse est également partagée par d'autres missions locales.

«On va identifier des problèmes lourds qui font que le jeune n'a finalement pas sa place dans la Garantie jeunes : il doit traiter ses problèmes psychiques, ses addictions, etc. avant. La conclusion à laquelle on arrive c'est : est-ce que la Garantie jeunes est la bonne réponse ?»

\section{(Directeur adjoint, ML C)}

Plus surprenant, car correspondant davantage à la figure classique de l'entreprise « usager du SPE », le registre du placement a également été tenu à distance des pratiques déployées par les missions locales. Les conseillers GJ sont restés souvent en retrait dans la relation directe à l'entreprise, estimant qu'il revenait aux jeunes, pour accéder à l'autonomie recherchée par le dispositif, de se mettre eux-mêmes en relation avec les entreprises. Le concept de médiation active, consistant comme on l'a vu plus haut à agir autant et de façon simultanée sur l'offre et la demande de travail, par exemple en négociant le profil du poste à pourvoir et en accompagnant l'employeur dans sa gestion des ressources humaines, a ainsi été d'abord et avant tout pensé en direction de l'accompagnement des jeunes, en amont donc de l'entreprise. Le travail des conseillers GJ, pour reprendre leur vocabulaire, a visé à « outiller les jeunes », à révéler leurs compétences, dont ils ignorent parfois jusqu'à l'existence, à leur faire découvrir des métiers en phase avec leurs univers et leurs valeurs de référence, puis à les conduire progressivement vers la construction d'un projet professionnel. En revanche, leur posture de travail n'a que peu consisté à équiper le marché du travail par une intervention auprès des entreprises, pour co-construire avec eux les conditions d'un rapprochement direct avec ces jeunes. Finalement, la médiation active telle qu'elle s'est traduite dans les faits n'a pas contribué à renforcer leur rôle d'intermédiaire entre les jeunes et les employeurs. À plusieurs reprises, la notion même d'intermédiaire a été rejetée par nos interlocuteurs :

« [Nous sommes] des médiateurs et non des intermédiaires. [...] ce n’est pas moi qui fait la mise en relation, elle se fait par le jeune, car c'est au jeune d'être acteur de sa recherche d'emploi, ce sont eux les ambassadeurs de la mission locale [...] Le jeune sait pourquoi il a envie de venir dans telle entreprise parce qu'on aura travaillé avec lui sur la valorisation de ses savoirs, savoir-faire et ses compétences transverses qu'il va pouvoir aller vendre directement auprès de l'entreprise. »

(Directrice adjointe, ML A)

C'est finalement l'objectif d'autonomisation, recherché par le dispositif, qui expliquerait la posture de retrait voire de « désintermédiation » (DE LARQUIER, TUCHSZIRER, 2017) affichée par les ML dans l'activité de rapprochement des jeunes et de l'entreprise. Au moins, cette vision de la médiation active comme abandonnant toute référence à un rôle de prospecteur-placier a-t-elle été effective. 
La difficulté à opérationnaliser le concept de médiation active a aussi pu résulter de l'absence d'une mise en discussion collective, quant au sens qu'il convenait de lui donner dans l'espace de la ML. Différents facteurs organisationnels (recrutements externes, pression temporelle, contraintes immobilières, etc.) déjà évoqués expliquent pourquoi la GJ a ainsi pu, dans ces premières vagues, se déployer à l'écart des autres équipes de conseillers et notamment à distance de l'expérience accumulée par les équipes emploi dans les années récentes (notamment via la mise en œuvre des emplois d'avenir). Dans certaines ML, les équipes « relations entreprises » et les équipes « GJ » travaillaient ainsi à partir de leur propre réseau d'entreprises partenaires pour des immersions et des mises à l'emploi. La ML C est dotée d'une équipe expérimentée de conseillers « entreprises », formés à la méthode IOD, l'une des sources théoriques et pratiques de la médiation active. Ces conseillers proposent une offre de services auprès des entreprises qui recrutent en emplois d'avenir, notamment des bilans réalisés dans l'entreprise, y compris avant la prise de poste, afin de sécuriser la relation de travail. L'équipe GJ, de son côté, est plus jeune et dispose de beaucoup moins d'expériences en termes de relations avec le monde de l'entreprise ; leur appréhension du marché du travail local est plus imprécise (LiMA, 2014). Une conseillère GJ d'une trentaine d'années, recrutée en interne, explique : «On n'a pas du tout une vision globale, analytique, de la situation du marché du travail, des secteurs porteurs. » « Ce n'est pas notre taf, c'est Pôle emploi qui publie les enquêtes de besoins de main-d'œuvre », complète un collègue du même âge, présent depuis huit ans dans la structure et ancien conseiller « emploi » avant de devenir conseiller Garantie jeunes.

Dans ces conditions, il a été malaisé de lever les ambiguïtés attachées à la notion complexe de la médiation active. Les équipes GJ ont par endroits davantage investi l'accompagnement du jeune, dans ses dimensions professionnelles, sociales ou pédagogiques, sans véritablement chercher à aller vers les employeurs, ceci d'autant plus que les jeunes étaient poussés à rechercher par eux-mêmes leurs terrains de stage. Les immersions s'apparentent alors à des expériences au coup par coup. Les employeurs sont vus comme offrant un service aux jeunes, mais les ML se mettent en position d'offrir un service aux employeurs dans peu de situations. Lorsque les entreprises acceptent de prendre en stage les jeunes, les conseillers de GJ expliquent cela par la dimension citoyenne de ces employeurs, qui peut tenir à leur fibre sociale ou parentale, ou de façon moins personnalisée à des engagements de responsabilité sociétale de l'entreprise. Ceci interroge la capacité de ces intermédiaires à entrer dans des rapports d'intéressement avec les employeurs qui viseraient à dépasser ce seul registre civique. Le principal support d'intéressement de la GJ porte ici sur la mise en forme de la qualité des jeunes. Au sein de la plus petite ML de l'échantillon, située en zone rurale, la dimension de proximité et l'étroitesse du territoire ont été évoquées premièrement comme des éléments favorisant la réussite de l'obtention d'immersions, plus que comme des facteurs d'insertion durable. Cette approche civique ou domestique a difficilement été combinée avec des intéressements marchands. La GJ ne propose pas d'aide financière aux employeurs. L'intéressement proposé, pour les entreprises, est 
donc hors coût. Dans cette perspective d'intéressement marchand, un usage pouvait être de coupler la GJ avec d'autres dispositifs (par exemple inciter à l'embauche via un emploi d'avenir ou un CIE-Starter), ou encore de valoriser l'intérêt économique pour l'employeur d'un pré-recrutement à peu de frais, les intermédiaires de l'insertion jouant alors dans ce partenariat le rôle de fournisseurs de main-d'œuvre (SEMENOwICZ, 2017). La dimension d'intéressement plus industrielle, calée sur une offre de services fondée sur des pré-embauches et des co-constructions de parcours de qualification, notamment dans des secteurs connaissant des difficultés de recrutement, dépend elle aussi de la qualité des réseaux locaux pour l'insertion. La mobilisation de l'offre de formation de la région ou encore une utilisation des contrats aidés conditionnée à des situations d'apprentissage sur le tas peuvent conduire à cet intéressement. Des exemples croisant ces formes industrielles et marchandes de l'intéressement ont été repérés, par exemple dans le cas d'une petite mission locale (treize salariés) située dans un bassin d'emploi (département C) avec un très faible taux de chômage et ayant construit un partenariat solide avec les entreprises industrielles et les nombreuses agences d'intérim locales. Le travail de mise en forme de qualités fait par la ML la rapproche d'ailleurs de ce que font les agences d'intérim quand elles certifient la qualité des profils de candidats avant de les placer sur une mission. L'accompagnement intensif des jeunes et la connaissance qu'en ont les conseillers peuvent ainsi constituer un élément de sécurisation des recrutements pour les entreprises. Tel a d'ailleurs été le point de vue de nombre d'établissements de travail temporaire qui se sont rapprochés des missions locales à l'occasion de la Garantie jeunes, élargissant pour ces dernières leur réseau de partenaires pour des immersions. Le service de relations « entreprises » de cette ML utilise par ailleurs la GJ comme un outil pour agir sur le recrutement. Une ergonome vient d'être recrutée pour travailler sur les fiches de poste avec les employeurs. Avec Pôle emploi, la ML a travaillé sur la création d'un SI-RH (système d'information RH) permettant de travailler sur les référentiels métiers et la montée en compétences des jeunes.

\section{La nouveauté de l'accompagnement collectif : la transformation de la relation aux jeunes}

C'est autour de l'accompagnement délivré aux jeunes que s'est construit un consensus sur le caractère particulièrement innovant de la GJ. Pour appréhender la dimension novatrice de cet accompagnement, nous nous appuyons sur deux définitions de l'innovation issues de travaux portant directement sur les jeunes et les missions locales, qui aident à qualifier la portée des transformations à l'œuvre. L'innovation contiendrait ainsi « deux notions qui sont à la fois l'idée de nouveau et l'idée de changement, toute la difficulté étant d'évaluer à quel moment on passe de la continuité à la rupture »(BARON et al., 1995). Bertrand Schwartz prolonge cette définition en insistant peut-être davantage sur la radicalité de l'innovation dans son rapport au changement. « Dans l'expérimentation, l'innovation ne peut se limiter à réparer, c'està-dire à recréer une situation antérieure dont on ne modifierait que quelques aspects mineurs. Ce qui importe, c'est de changer la situation, de la transformer, de faire en 
sorte que le retour au point initial devienne de plus en plus difficile » (SCHWARTZ, SARAZIN, 2006). L'accompagnement collectif tel qu'il s'est mis en place dans les ML relève sans aucun doute de ces deux définitions de l'innovation et remplit même la condition de rupture qu'évoquent, chacun à leur façon, les auteurs.

L'accompagnement collectif, ainsi que le travail en binôme (deux conseillers agissant en binôme pour chaque cohorte) ont constitué les deux principaux changements par rapport au référentiel classique des ML, centré sur des entretiens individuels plus échelonnés dans le temps. Avec la GJ, les jeunes sont suivis durant les premières semaines du contrat d'un an, de façon intensive, tous les jours et à temps plein. Ce rythme de travail vient rappeler celui de l'entreprise. De même, le travail en groupe réalisé dans le cadre des promotions, rassemblant en moyenne une douzaine de jeunes, place ces derniers dans des situations d'interaction qui les préparent à la vie de l'entreprise.

Cette nouvelle relation aux jeunes portée par ces moments collectifs a été fortement valorisée. L'idée que la GJ vient transformer en profondeur les modalités d'accompagnement des jeunes fréquentant les ML est une analyse partagée, de l'équipe de direction aux conseillers, qu'ils aient été ou non dédiés à ce dispositif. Deux responsables nous ont parlé du "vernis » derrière lequel les jeunes peuvent se cacher en entretien individuel ; or en groupe « on gratte et le vernis tombe rapidement » (directrice MLA). « On peut déceler des problèmes graves d'ordre cognitif, physique, d'addiction, etc., qui passaient inaperçus dans le cadre du suivi classique », explique ainsi une conseillère GJ de la ML B. L'idée est aussi que cette meilleure connaissance permet d'être plus efficace dans l'orientation et la prescription d'outils. « On sent plus rapidement sur quoi il va falloir mettre la priorité » (conseillère GJ, ML B). Certains outils comme la méthode des compétences fortes, évoquée plus haut, mais aussi les immersions en entreprise quand les conseillers ont le temps d'en faire le bilan avec les jeunes, sont appréciés en ce qu'ils permettent de remobiliser les jeunes, de leur faire prendre conscience de leurs talents, de leur redonner confiance en eux, de les «booster» vers une démarche plus dynamique ${ }^{16}$.

« Dans le groupe on leur explique qu'on est tous dans le même bateau, on n'est pas là pour les dévaloriser, parce que bien souvent ils manquent de confiance, ils sont peu valorisés. L'objectif, c'est que, mieux ils vont se connaître, mieux derrière ça va être facile de les accompagner, mieux ils vont savoir sur quoi ils ont des difficultés, mais surtout sur quoi ils ont des points forts. On leur a tellement dit qu'ils étaient nuls, bons à rien, bon je caricature, que du coup plus on va leur dire : "ça, tu sais faire", ça les valorise. Mieux ils vont se connaître, mieux derrière l'orientation professionnelle mais aussi l'insertion professionnelle va être facilitée, parce qu'ils vont pouvoir dire

16. Cette remobilisation psychologique des jeunes dans la recherche d'emploi - proche d'une logique de coaching - est ainsi très souvent vue comme une condition de leur capacité à se présenter devant un employeur et de tenir un emploi, mais il s'agit d'une intervention exclusivement centrée sur le versant individuel de la recherche d'emploi (BoNvIN et al., 2013 ; MAzouz, 2014). 
à un entretien d'embauche "ça je sais faire" ou "ça je ne sais pas faire mais je peux apprendre". »

\section{(Conseillère GJ, ML C, 33 ans, diplôme de travail social, ex-formatrice, vient d'être recrutée)}

Les conseillers associent souvent le chômage des jeunes à des difficultés individuelles. Cette démarche clinique confine alors parfois à une certaine psychologisation des relations. Dans l'équipe GJ de la ML C, un conseiller évoque l'idée que « la connaissance de leur intimité s'impose à nous », ce qui est valorisé par les conseillers, dans le sens où cela permet une meilleure relation. Dans leurs relations avec des employeurs potentiels, cette meilleure connaissance permettrait ainsi de se poser en garant des qualités individuelles et donc en intermédiaire de confiance.

Toutefois, la focalisation dont a fait l'objet cette première phase de l'accompagnement, centrée sur l'accueil de cohortes, a eu pour effet de négliger l'autre partie de l'accompagnement, celle moins spectaculaire mais tout autant attendue, relative au suivi des jeunes au-delà des premières semaines d'accompagnement collectif. Autant l'accompagnement en groupe a fait l'objet d'une pensée, d'une planification, d'une gestion du temps (succession de cohortes sur un temps rapproché) et de l'espace (location de nouveaux locaux) pour accueillir ces promotions de jeunes, autant le suivi individuel de ces mêmes jeunes, une fois passé les premières semaines d'accompagnement, est resté du domaine de l'impensé. Les conseillers GJ doivent s'adapter à des flux mal maîtrisés de sollicitations directes des jeunes. L'innovation qu'a constituée l'introduction de l'accompagnement collectif a ainsi sa part d'ombre. Si ce nouveau référentiel a cristallisé nombre des enjeux associés à ce dispositif, sa mise en œuvre a été source de bouleversements organisationnels majeurs, au point d'avoir pu occulter l'autre enjeu que constituait le suivi au long cours des jeunes durant les dix à onze mois suivants. C'est un point sensible auquel les ML tentent de remédier pour penser l'accompagnement dans toute sa durée. Une autre occultation peut tenir aux effets de focalisation qu'a exercés la GJ : bien que ne concernant qu'une minorité des jeunes au sein des ML, ce dispositif a concentré les regards externes (les acteurs locaux et nationaux, la presse) mais aussi internes, au risque de dévaloriser le travail réalisé par les autres conseillers.

Enfin, dans cet accompagnement repensé, la place de l'allocation monétaire aurait pu faire débat et donner lieu à des tensions importantes dans le travail. Or la plupart des conseillers n'identifient pas la présence de cette allocation monétaire comme un problème : « elle est là dès le début » dit une conseillère GJ. On notera qu'une partie des conseillers ont été recrutés depuis l'extérieur pour gérer ce dispositif : il n'y a pas pour eux d'évolution de leurs pratiques. De leur côté, les conseillers qui étaient déjà dans les ML avant la GJ ont expérimenté depuis une quinzaine d'années divers dispositifs d'allocation financière reposant justement sur un suivi renforcé et orienté « emploi ». L'idée selon laquelle la présence d'une allocation monétaire viendrait dénaturer la relation d'insertion avec les jeunes (FARVAQUE, BENLEMSELMI, 2003) n'est 
plus de mise et les conseillers rencontrés plaident au contraire bien pour l'existence d'un tel outil de nature à solder - temporairement - des situations de précarité financière (LOISON-LERUSTE et al., 2016), de façon à pouvoir véritablement placer les jeunes dans une dynamique d'insertion.

\section{Conclusion}

La Garantie jeunes a été bien accueillie par les missions locales qui sont apparues en phase avec les objectifs portés par ce dispositif. Cette adhésion aux objectifs ne signifie pas pour autant que la mise en œuvre de la GJ ait permis de les atteindre tous simultanément. Il ressort de ces différentes observations que si la relation aux jeunes a été transformée sous l'effet de la GJ, la relation aux entreprises n'a, elle, pas été modifiée en profondeur. La GJ a d'abord été pensée comme un dispositif d'accompagnement des jeunes plus que de médiation envers les entreprises. Par ailleurs, la référence à l'autonomie des jeunes voulue par le dispositif a fait l'objet d'une traduction opérationnelle particulière de la part des missions locales, en décalage, sans doute, avec le principe de la médiation active qui supposait pour les conseillers d'entrer en négociation avec les entreprises pour en favoriser l'accès aux jeunes. Enfin, la catégorie des Neets vulnérables et précaires qu'il s'agissait de repérer dans le cadre de ce dispositif s'est révélée d'une faible portée opérationnelle. Les ML font partie de l'univers institutionnel de ces jeunes qui ont pu, à un moment donné de leur existence, fréquenter de telles structures. La question posée est semble-t-il moins celle du repérage de ces jeunes que de leur mobilisation effective dans le dispositif. Toutefois, il est à souligner que nos observations sont intervenues à un moment bien spécifique de l'évaluation, très peu de temps après le lancement de l'expérimentation et bien avant sa généralisation en 2017. Seules les premières vagues de l'expérimentation ont été ici examinées, ce qui invite à la prudence quant à la généralisation de nos propres observations. Ces réserves émises, on peut néanmoins souligner à quel point le modèle d'accompagnement des jeunes amorcé dans le cadre de la GJ semble apporter une réelle plus-value dans la façon de suivre ces jeunes. Le travail collectif réalisé par un binôme de conseillers avec des cohortes de jeunes mobilisés quotidiennement durant les premières semaines du dispositif renouvelle incontestablement la façon de penser l'accompagnement dans les politiques de l'emploi. C'est une forme d'accompagnement qui s'est avérée, à l'usage, exigeante puisqu'elle a impliqué un engagement important à la fois des conseillers et des personnes suivies.

Au-delà de ces résultats, nous souhaiterions conclure sur le caractère expérimental de ce dispositif qui vient appuyer d'autres constats et recherches ayant mis en évidence le rapport ambigu que l'État central entretient avec les acteurs locaux pour renouveler l'action publique (BUREAU et al., 2013). Ceci permet de resituer nos observations dans le cadre plus large d'une sociologie de la mise en œuvre de l'action publique. Celle-ci se réfère à des volontés d'expérimentation tout en cadrant relativement fortement - ou 
du moins au début, pour les premiers territoires pilotes - les moyens et les finalités de l'action en matière d'insertion. L'État organise le changement au sein des ML, structures indépendantes et enracinées localement, de façon nécessairement indirecte, par le biais d'injonctions à respecter dans le cadre de dispositifs nationaux.

Avec la GJ, s'est-il agi de faire appel aux capacités d'initiatives et d'innovations des missions locales pour déployer une nouvelle forme d'intervention sociale en direction des jeunes ? Ne peut-on pas plutôt considérer, au vu des modalités de déploiement de la GJ, que l'expérimentation s'est surtout apparentée à une phase de test, une sorte de pilote qui allait précéder une généralisation du dispositif déjà actée dans l'esprit des ML ? Les missions locales de ces premières vagues de mise en œuvre, observées dans le cadre de l'évaluation, se sont incontestablement placées dans cette hypothèse. Elles ont donc respecté scrupuleusement les consignes et recommandations formulées par l'État central et ses échelons déconcentrés (préfet, Direccte) en s'accordant peu de degrés de liberté dans l'application du dispositif.

On a pu constater, pour les missions locales qui ont expérimenté le dispositif plus tardivement, peu de temps avant la généralisation de la GJ, qu'il en a été autrement. Une fois la pression politique et médiatique retombée, les ML des dernières vagues ont pu tirer un plus grand bénéfice du cadre expérimental proposé. Elles ont pris leur temps pour inscrire la GJ dans une logique territoriale fédérant ainsi l'ensemble des conseillers autour des projets retenus. L'État, de son côté, a pu également tirer de ces espaces d'innovation que sont les missions locales des enseignements importants avant de généraliser la GJ. C'est peut-être dans cette voie, celle d'un dialogue territorial renforcé entre l'État central et les acteurs locaux, qu'il convient de poursuivre pour renouveler le cadre de l'action publique dans le champ des politiques d'insertion.

\section{BIBLIOGRAPHIE}

Astier I. (1997), Revenu minimum et souci d'insertion, Paris, Desclée de Brouwer.

Baron C., Bureau M.-C., Le Dantec E., Nivolle P. (1994), Les Intermédiaires de l'insertion, Paris, CEE, Dossier $n^{\circ} 1$, nouvelle série.

BARBIER J.-C., Eymard-Duvernay F. (1996), « Gestion de l'emploi dans les entreprises et politiques publiques », 4 pages du Centre d'études de l'emploi, $\mathrm{n}^{\circ} 15$.

Baron C., Bureau M.-C., Gomel B., Leymarie C., Nivolle P., Schmidt N., Wissler A., (2005), «Les missions locales et l'innovation », Rapport de recherche, CEE, $\mathrm{n}^{\circ} 21$.

Baron C., Bureau M.-C., Leymarie C., Nivolle P. (1995), « La construction de réseaux locaux pour l'insertion et la qualification : l'exemple d'un GEIQ » in Les Politiques publiques d'emploi et leurs acteurs : des repères pour l'évaluation, Cahiers du CEE, nº 34, pp. 213-233.

BERHUET S. (2013), Les Chômeurs et les intermédiaires de l'emploi : une sociologie dynamique de leurs trajectoires au sein d'une Maison de l'emploi, Thèse de doctorat en sociologie, Cnam. 
Boltanski L., ThéVenot L. (1991), De la justification. Les économies de la grandeur, Paris, Gallimard.

Bonvin J.-M., Dif-Pradalier M., Rosenstein E. (2013), « Politiques d'activation des jeunes et modalités d'accompagnement. Le cas du programme FORJAD en Suisse », Lien social et politiques, $\mathrm{n}^{\mathrm{o}} 70, \mathrm{pp} .13-27$.

Bureau M.-C., Marchal E., (2009), «Incertitudes et médiations au cœur du marché du travail », Revue française de sociologie, vol. 50, $\mathrm{n}^{\circ} 3$, pp. 573-598.

Bureau, M., Sarfati, F., Simha, J., TuchSzIRER, C. (2013), « L'expérimentation dans l'action publique : usages, pratiques et jugements des acteurs », Travail et Emploi, n ${ }^{\circ} 135$, pp. 41-55.

Clasquin B., Meyer J.-L., Charlier M., Lioger R. (2000), « Agences locales pour l'emploi, missions locales et PAIO dans trois zones d'emploi en Lorraine : des injonctions nationales aux pratiques locales », in Gélot D., Nivolle P. (dir.), Les Intermédiaires des politiques de l'emploi, Paris, La Documentation française.

De Larquier G., TuchsZirer C. (2017), « De l'impératif du placement à la désintermédiation du marché du travail. Évolution du modèle d'intermédiation du Service public de l'emploi », $37^{\mathrm{e}}$ Journées de l'AES, université Paris 8.

Dubois V. (2010), La Vie au guichet. Relation administrative et traitement de la misère, Paris, Economica.

EYMARD-DuVERnAY F., MARCHAL E. (1997), Façons de recruter : le jugement des compétences sur le marché du travail, Paris, Métailié.

EYMARD-DuvernAY F., MARCHAL É. (1994), « Les règles en action : entre une organisation et ses usagers », Revue française de sociologie, vol. 35, n 1, pp. 5-36.

FARVAQUE N. (2004), «L'encadrement des acteurs locaux de l'insertion des jeunes en France », Les Politiques sociales, $\mathrm{n}^{\circ} 1-2$, pp. 108-125.

FARvaque N., Benlemselmi M. (2003), « La relation d'insertion "travestie" ? Règles et conventions d'action publique face à la mise en œuvre de la Bourse d'accès à l'emploi » in Euzéby C. et al. (dir.), Mondialisation et régulation sociale, XXIII Journées de l'AES, Paris, L'Harmattan, pp. 809-823.

Farvaque N., Kramme C., Tuchszirer C. (2016), La Garantie jeunes du point de vue des missions locales : un modèle d'accompagnement innovant, mais source de bouleversements organisationnels, rapport d'évaluation pour la DARES.

Farvaque N., Curabet J., SÉBAstien A. (2017), Les Partenariats entre missions locales et entreprises, au centre de l'accompagnement des jeunes en emplois d'avenir, Étude réalisée pour la Direccte Île-de-France.

FARVAQue N., MessaOUdi D. (2013), Enquêtes monographiques sur les premiers mois de mise en œuvre des Emplois d'avenir, rapport pour la DARES.

Fretel A., Pillon J.-M., Remillon D., Tuchszirer C., Vivés C., Fondeur Y. (2016), « Dynamiques territorialisées du champ de l'intermédiation », in Diversité et dynamiques des intermédiaires du marché du travail, Pôle emploi, Études et Recherches $\mathrm{n}^{\circ}$ 7, pp. 5-162. 
GARRAud P. (2000), Le Chômage et l'action publique. Le bricolage institutionnalisé, Paris, L'Harmattan.

Gouzien, A. (2009), « Pénurie de main-d'œuvre et dispositifs localisés d'insertion dans les pêches maritimes en Bretagne », Travail et Emploi, $\mathrm{n}^{\circ}$ 119, pp. 23-35.

Hill M., HuPE P. (2002), Implementing public policy: governance in theory and practice, London, Sage.

IGAS (2016), Le Modèle économique des missions locales pour l'insertion professionnelle et sociale des jeunes, Rapport public.

KARPIK L. (1996), « Dispositifs de confiance et engagements crédibles », Sociologie du travail, vol. $38, \mathrm{n}^{\circ} 4$, pp. 527-550.

Kretzschmar C., Michiels M-A. (2000), Trajet d'accès à l'emploi en Rhône-Alpes, évaluation partagée de sa mise en œuvre, Ministère de l'Emploi et de la Solidarité, DRTEFP Rhône-Alpes, Rapport d'évaluation.

LATOuR B. (2003), «L'impossible métier de l'innovation technique - PROTEE une nouvelle méthode d'évaluation des projets fortement incertains », in Mustar P., Penan H. (dir.), Encyclopédie de l'innovation, Paris, Economica, pp. 9-26.

LiMA L. (2014), « Qui recrute et comment? L'expertise économique des professionnels de l'insertion des jeunes », Sociologie du travail, vol. 56, n 2, pp. 161-181.

Lima L. (2016), Pauvres jeunes. Enquête au cœur de la politique sociale de jeunesse, Nîmes, Éditions Champ social.

Loison-Leruste M., Couronné J., F. SARfati (2016), La Garantie jeunes en action. Usages du dispositif et parcours de jeunes, Rapport pour la DARES.

MAS S. (2002), «Un jeune sur deux en emploi à la sortie du dispositif TRACE », Premières Informations et Premières Synthèses, $\mathrm{n}^{\circ} 34.1$, DARES.

Mazouz, S. (2014), « Le cadre de l'émancipation. Se conformer à l'offre d'emploi dans une mission locale », Politix, vol. 108, n 4, pp. 31-52.

MEYer J.-L. (1998), « Intermédiaires de l'emploi et marché du travail », Sociologie du travail, vol. 40, n 3, pp. 345-364.

Muniglia V., Thalineau A. (2012), « Insertion professionnelle et sociale des jeunes vulnérables. Les conseillers des missions locales entre adaptation et tension », Politiques sociales et familiales, $\mathrm{n}^{\mathrm{o}}$ 108, pp. 73-82.

Roche P. (2007), « Les défis de la proximité dans le champ professionnel », Nouvelle revue de psychosociologie, vol. $1, \mathrm{n}^{\mathrm{o}} 3$, pp. 63-82.

SABATIER P. A. (1986), "Top down and bottom up approaches to implementation research", Journal of Public Policy, vol. 6, n ${ }^{\circ}$ 1, pp. 21-48.

Sabatier P. A., Mazmanian D. A. (1979), "The Conditions of Effective Implementation", Policy Analysis, vol. 5, $\mathrm{n}^{\circ}$ 4, pp. 481-504. 
SALOGNON M. (2005), «Infléchir les comportements d'embauche des entreprises : l'exemple de la méthode IOD », in Problèmes politiques et sociaux sur «Les jeunes non qualifiés », La Documentation française, $\mathrm{n}^{\circ}$ 915, pp. 89-90.

SchWARTz B. (1994), Moderniser sans exclure, Paris, La Découverte.

Schwartz B., SARAzin G. (2006), « La recherche-action collective », Dossier « Construire une pensée collective pour l'action », Revue POUR, Grep, nº 89.

Semenowicz P. (2017), Collaborer pour insérer? Les partenariats sociaux dans l'insertion par l'activité économique, Rennes, Presses universitaires de Rennes.

WRIGHT, S. (2002), "Activating the unemployed: the street-level implementation of UK policy", Tijdschrift voor Arbeid en Participatie, vol. 24, n ${ }^{\circ}$ 2, pp. 105-124.

WuHL S. (1996), Insertion : les politiques en crise, Paris, Presses universitaires de France.

\section{ANNEXE - SYNTHÈSE DES ENTRETIENS RÉALISÉS}

\begin{tabular}{|c|c|c|c|}
\hline & MLA & ML B & ML C \\
\hline Direction ML & 2 & 2 & 2 \\
\hline Conseillers GJ & 2 & 2 & 7 \\
\hline Conseillers insertion & 2 & 1 & 1 \\
\hline Conseillers emploi/CRE & 1 & 1 & 2 \\
\hline Autres conseillers ML & & & 1 \\
\hline Préfecture & 1 & & \\
\hline Direccte & 1 & 2 & 2 \\
\hline Pôle emploi & & 1 & 1 \\
\hline Éducation nationale & & 1 & \\
\hline SPIP & & 1 & \\
\hline PJJ & & 1 & \\
\hline Région & & & 1 \\
\hline Conseil départemental & & 1 & 2 \\
\hline Jeunesse et Sport & & & 1 \\
\hline Caf & & 1 & \\
\hline Afpa & & & 1 \\
\hline Cap emploi & & & 1 \\
\hline Associations (prévention), foyer hébergement & 1 & & 1 \\
\hline CCI, Chambre des métiers, Maison de l'emploi et de la formation & 1 & & 1 \\
\hline Commission locale & $\mathrm{X}$ & $\mathrm{X}$ & $\mathrm{X}$ \\
\hline Commission départementale & $\mathrm{X}$ & $\mathrm{X}$ & $\mathrm{X}$ \\
\hline Observation atelier GJ & $\mathrm{X}$ & $\mathrm{X}$ & $\mathrm{X}$ \\
\hline Autres ML du département & 1 (1 ML) & $2(1 \mathrm{ML})$ & 3 (3 ML) \\
\hline $\begin{array}{l}\text { Autres entretiens et observations participantes } \\
\text { Responsables DGEFP } \\
\text { UNML } \\
\text { ANDML } \\
\text { Comité de pilotage national GJ décembre } 2015 \\
\text { Séminaire européen ( } \text { Peer Review »), avril } 2016 \\
\text { Séminaire d'échanges de pratiques, conseillers et directeurs } \\
\text { des ML du département de ML C, mars } 2016\end{array}$ & & & \\
\hline
\end{tabular}

